\title{
Factors associated with the decision to undergo risk-reducing salpingo-oophorectomy among women at high risk for hereditary breast and ovarian cancer: a systematic review
}

\author{
Sun-young Park ${ }^{1,2}$, Youlim Kim ${ }^{1,3}$, Sue Kim ${ }^{3}$ \\ ${ }^{1}$ Graduate School, Yonsei University, Seoul, Korea \\ ${ }^{2}$ National Evidence-based Healthcare Collaborating Agency, Seoul, Korea \\ ${ }^{3}$ Mo-Im Kim Nursing Research Institute and College of Nursing, Yonsei University, Seoul, Korea
}

Purpose: This systematic review aims to identify factors associated with risk-reducing salpingo-oophorectomy (RRSO), including the uptake rate and decision timing, among women at high risk for hereditary breast and ovarian cancer (HBOC).

Methods: We found 4,935 relevant studies using MEDLINE, Embase, CINAHL, and PsycINFO on July 6, 2020. Two authors screened the articles and extracted data. Twenty-four studies met the inclusion criteria. Quality assessment of articles was conducted using the Risk of Bias for Nonrandomized Studies tool.

Results: Five types of factors were identified (demographic factors, clinical factors, family history of cancer, psychological factors, and objective cancer risk). The specific significant factors were older age, having child(ren), being a $B R C A 1 / 2$ carrier, mastectomy history, perceived risk for ovarian cancer, and perceived advantages of RRSO, whereas objective cancer risk was not significant. The uptake rate of RRSO was $23.4 \%$ to $87.2 \%$ (mean, $45.2 \%$ ) among high-risk women for HBOC. The mean time to decide whether to undergo RRSO after BRCA testing was 4 to 34 months.

Conclusion: RRSO decisions are affected by demographic, clinical, and psychological factors, rather than objective cancer risk. Nonetheless, women seeking RRSO should be offered information about objective cancer risk. Even though decision-making for RRSO is a complex and multifaceted process, the psychosocial factors that may influence decisions have not been comprehensively examined, including family attitudes toward RRSO, cultural norms, social values, and health care providers' attitudes.

Keywords: Decision making; Hereditary breast and ovarian cancer syndrome; Salpingo-oophorectomy; Systematic review

Received: October 12, 2020

Revised: November 18, 2020

Accepted: November 19, 2020

Corresponding author:

Sue Kim

College of Nursing, Yonsei

University, 50-1 Yonsei-ro,

Seodaemun-gu, Seoul 03722, Korea

Tel: +82-2228-3276

E-mail: suekim@yuhs.ac

\section{Introduction}

Hereditary breast and ovarian cancer (HBOC) is a syndrome that is associated with an increased incidence of breast and ovarian cancers [1]. Approximately $12 \%$ of breast cancer and $1 \%$ to
$2 \%$ of ovarian cancer cases occur in women with HBOC [2]. The most common causes of HBOC are mutations in the breast cancer susceptibility 1 and 2 genes (BRCA1 and BRCA2, respectively) $[1,2]$. BRCA mutations are found in $15.7 \%$ of women with a personal or family history of breast/ovarian cancer in

This is an Open Access article distributed under the terms of the Creative Commons Attribution License (http://creativecommons.org/licenses/by/4.0) which permits unrestricted use, distribution, and reproduction in any medium, provided the original work is properly cited.

Copyright (c) 2020 Korean Society of Women Health Nursing 


\section{Summary statement}

\section{- What is already known about this topic?}

Decision-making for risk-reducing salpingo-oophorectomy (RRSO) in women at high risk for hereditary breast ovarian cancer (HBOC) is a complex process influenced by demographic, clinical, psychological factors, and family history of cancer.

\section{- What this paper adds}

Significant factors for RRSO were older age, having child(ren), being a BRCA carrier, mastectomy history, perceived risk of ovarian cancer, and perceived advantages of RRSO. Objective cancer risk, however, was not significant.

- Implications for practice, education, and/or policy

Psychosocial factors should be comprehensively examined for women at high risk for HBOC contemplating RRSO, including family attitudes, cultural/social values, and health care providers' attitudes. Women seeking RRSO should be offered information about their objective cancer risk.

Korea [3]. Among BRCA1 carriers, the average lifetime cancer risks are $67 \%$ for breast cancer and $45 \%$ for ovarian cancer. Among BRCA2 carriers, these risks are $66 \%$ and $12 \%$ for breast and ovarian cancer, respectively. Therefore, clinical prevention options, such as risk-reducing salpingo-oophorectomy (RRSO), risk-reducing mastectomy (RRM), intensive surveillance for early detection of ovarian/breast cancer, and chemoprevention with tamoxifen, are offered to women at high risk for HBOC $[1,2]$.

Among these preventive strategies, the most frequently offered option is RRSO because it reduces the risk of cancer incidence by more than $95 \%$ for ovarian cancer and $50 \%$ for breast cancer [4]. The National Comprehensive Cancer Network recommends that RRSO should be considered for women with $B R C A$ mutations aged 35 to 40 years who have completed childbirth [5]. However, deciding to undergo RRSO is not an easy process and is affected by multifaceted factors $[1,5,6]$. Because RRSO not only causes postoperative menopause $[1,5]$, but also negatively affects quality of life and psychological health, and can also alter one's identity as a woman $[5,7]$. Previous studies have shown that women tend to overestimate their own cancer risk [8], to perceive information about cancer risk and preventive surgery as lacking [9], and to feel that there is pressure from clinical specialists to choose certain preventive options [10]. Therefore, genetic counseling should be improved to provide helpful decision-making support, and to do so, it is necessary to understand the current process of making decisions about RRSO $[5,7]$.

After disclosure of the results of the BRCA test, it takes a long time for some women to select a preventive option [7], and RRSO rates have been reported to range from $13 \%$ in the USA to $75 \%$ in the Netherlands [11]. Therefore, a comprehensive ex- ploration of the rate and timing of decision-making regarding RRSO is needed.

Although previous studies have shown that RRSO decision-making is influenced by demographic, clinical, psychological, and social factors [7,11-13], only one systematic review has integrated and explored various factors, and it did not identify significant factors [7]. Furthermore, perceptions of HBOC and RRSO, as well as healthcare infrastructure and culture, have changed since that systematic review was published in 2009 [7]. In Korea, the Health Insurance Review and Assessment Service approved BRCA1/2 tests and RRSO for high-risk populations for HBOC in 2012 and 2013, respectively [14]. In addition, international interest in $B R C A$ and preventive surgery has in creased since the actress Angelina Jolie, who is a BRCA1 carrier, received RRM in 2013 [13,14]. Despite prior research, there are gaps in explaining the process and factors associated with current RRSO decision-making.

This systematic review sought to identify the factors that influence the decision to undergo RRSO based on the existing literature. In addition, we explored the uptake rate of RRSO and the time interval between $B R C A$ testing and RRSO among women at high risk for HBOC.

\section{Methods}

Ethics statement: This study is a literature review of previously published studies and was therefore exempt from institutional review board approval.

This systematic review was conducted in accordance with the guidelines proposed by the Preferred Reporting Items of Sys- 
tematic Review and Meta-Analysis (PRISMA) [15]. The study protocol was registered prospectively at the National Institute for Health Research (registration number: CRD42020188202).

\section{Literature search}

To decide on the search terms, we reviewed 100 abstracts from relevant studies retrieved from MEDLINE. We combined keywords, such as "hereditary breast ovarian cancer," "BRCA," "risk-reducing surgery," and "decision-making," using "OR" for similar concepts and using "AND" for differing clusters. On July 6, 2020, the MEDLINE, Embase, CINAHL, and PsycINFO databases were searched, without any limitations on publication date (Supplementary Table 1).

\section{Study selection criteria}

To identify suitable studies for this study purpose, PICO (population, intervention, comparison, outcome) criteria were defined and listed (Table 1). Based on PICO, the inclusion criteria were determined as follows: studies that (1) were about women at high risk for HBOC (e.g., BRCA1/2 carriers, women with a family history of breast and/or ovarian cancers in multiple generations, and women who received genetic counseling for [risk of] breast and/or ovarian cancers); (2) reported on the factors influencing RRSO decision-making; and (3) were written in Korean or English. The exclusion criteria were as follows: (1) gray literature without peer review (e.g., conference abstracts, dissertations, and white reports); (2) animal experiments or preclinical experiments; (3) reviews, letters, and editorials; and (4) qualitative research.

Duplicate studies and gray literature were removed using a bibliography management program (EndNote X7, Clarivate, London, UK). Two authors (SYP and YLK) independently reviewed the titles and abstracts of identified studies, and selected studies according to the selection criteria.

\section{Risk-of-bias assessment}

Two authors (SYP and YLK) independently assessed the quality of selected articles using the Risk of Bias for Nonrandomized Studies tool (RoBANS, version 2.0) [16]. RoBANS is a risk-ofbias tool for non-randomized trials (e.g., cohort studies, case-control, and before-and-after studies) that evaluates the selection of populations, confounding variables, measurement exposure, outcome blinding, incomplete data, and selective reporting. RoBANS has moderate reliability, acceptable validity, and is compatible with domains of the Cochrane risk-of-bias tool [16]. The results of evaluating these domains were presented as "low," "high," and "unclear" with Revman (version 5.0, Cochrane Community, Oxford, UK).

\section{Data analysis}

One author (SYP) extracted the data from the selected literature using a predesigned form (first author and publication year, research country, population characteristics, study design and time of measurement, measurement, and significant or insignificant factors influencing RRSO), and another author (YLK) cross-checked the accuracy of data extraction. To show the overall significance of the reported factors, we synthesized data if the relevant factors were reported in two or more studies, and presented the ratio of the number of papers between significant and non-significant factors. The factors affecting decisions about RRSO were categorized into (1) demographic factors, (2) clinical factors, (3) family history of ovarian/breast cancer, (4) psychological factors, and (5) the objective risk of developing ovarian or breast cancer. To analyze the RRSO uptake rate, the intent and rate of RRSO were presented separately.

Table 1. PICO framework

\begin{tabular}{ll}
\hline Population $(\mathrm{P})$ & Women at high risk for HBOC include the following: \\
& - BRCA1/2 carriers \\
& - Women with a family history of breast and/or ovarian cancers in multiple generations \\
Intervention (I) & Studies that analyzed factors associated with RRSO decision-making \\
Comparators (C) & Factors associated with decision-making of the following: \\
& - Chemoprevention \\
& - Surveillance \\
& - Risk-reducing mastectomy \\
& Factors associated with RRSO decision-making among women at high risk for HBOC \\
Outcomes (O) & Rates of RRSO decision-making \\
& Timing of RRSO decision-making \\
\hline
\end{tabular}

BRCA: Breast cancer susceptibility gene; HBOC: hereditary breast and ovarian cancer; RRSO: risk-reducing salpingo-oophorectomy. 


\section{Results}

\section{Characteristics of the selected studies}

The chosen search strategies identified a total of 4,935 studies, from which 24 studies were finally included in the systematic review [11-14,17-36] (Table 2, Figure 1). The 24 studies included a total of 6,793 women (range, 42-1,241), and 10 were conducted in the United States, nine in Europe, two in Korea, and one each in Australia, Israel, and the United States/Canada/Europe together. Five studies (20.8\%) were cross-sectional, 9 (37.5\%) were prospective cohorts, and 10 (41.7\%) used a retrospective cohort design. The selected literature were published between 1999 and 2019.

\section{Risk-of-bias assessment}

The risk of bias in the selected literature was moderately low (Figure 2). In particular, four domains were assessed as having a low risk of bias: selection of the population (91.7\%), confounding variables $(87.5 \%)$, measurement of exposure (100\%), and incomplete data reporting $(95.8 \%)$. For the blinding of the outcome evaluation, the risk of bias was unclear in $41.7 \%$ of articles, and for the section of selective reporting, $25 \%$ of articles were evaluated as unclear because they did not report whether the institutional review board had approved their research protocol or whether the researchers prospectively conducted their study after the protocol was registered. Although it was difficult to determine the level of bias of these two domains, we assumed that these two domains did not significantly affect the overall quality of the selected articles. Thus, all selected studies were included in the data analysis (Figure 2).

\section{Factors associated with RRSO decision-making}

\section{Demographic factors}

Among the 19 studies that explored the effect of age on RRSO, $13(68.4 \%)$ reported that older age was associated with a higher uptake of RRSO [11-13,21,23-26,28,30,31,33,35]. A Korean study [12] showed more instances of RRSO in women in their 40 s than in their 50s, but the RRSO rate was higher in those over 50 years old in the United States [13] and France [25].

In $60.0 \%$ of the articles $[11,23,26]$, more women chose RRSO if they had a child(ren). Marital status (80.0\%) [20,29,32,33], employment status (100\%) [12,14,20,32], education level (88.9\%) [12-14,20,21,30,32,33], and race (80.0\%) [13,20,21,32] were consistently non-significant factors for RRSO.

\section{Clinical factors}

All of the articles (100\%) found that BRCA1/2 gene mutations were a significant factor in decision-making about RRSO $[5,19,23,30]$; however, the type of BRCA mutation was not a significant factor in the articles $(83.3 \%)$ that reported it $[11,13$, $14,26,28]$. A personal history of mastectomy was a significant factor in $80.0 \%$ of articles $[13,18,22,27]$, while only $40.0 \%[12,23,27]$ and $33.3 \%$ of articles $[14,23]$ reported that a personal history of breast cancer and menopausal status were significant factors for RRSO.

\section{Family history of cancer}

The vast majority of articles (81.8\%) [12-14,20-22,28,32,36] reported that a family history of breast cancer influenced RRSO decision-making; however, a family history of ovarian cancer was not a significant factor in $66.6 \%$ of articles [12-14,19,20, 22,33,36] (Tables 2, 3). Singh et al. [13] particularly suggested that although a family history of ovarian cancer was not a significant factor, the death of a mother or relative from pelvic or breast cancer affected RRSO decisions.

\section{Psychological factors}

The perceived risk/anxiety/concern for ovarian cancer was a significant factor in determining RRSO in most studies $(91.7 \%$, 11 of 12) that reported it [18-21,29,31-36]. Meanwhile, the perceived risk for breast cancer was not significant in two articles $[21,32]$. Four articles $[20,29,33,34]$ consistently reported that positive perceptions of RRSO were related to the decision to undergo surgery. With regard to negative perceptions of RRSO, two studies $[20,34]$ reported conflicting results. Cancer-related distress $[20,21]$, anxiety $[33,34]$, and depression $[12,33]$ were not significant factors for RRSO in the two articles that reported those factors. The significance of health perceptions differed between the two studies $[12,29]$.

\section{Objective cancer risk}

As a possible factor influencing RSSO decision-making, the reported objective cancer risk was the risk level evaluated by family cancer/genetic specialists based on a person's family cancer history [34,36], and breast cancer risk assessment tool according to the person's cancer status and family cancer history such as the BRCAPRO statistical model $[20,21]$. The objective cancer risk did not influence women's RRSO decision-making in four articles [20,21,34,36].

\section{Rate and timing of RRSO decision-making}

The RRSO rate was $11 \%$ to $87.2 \%$ across the 21 articles. In six stud- 


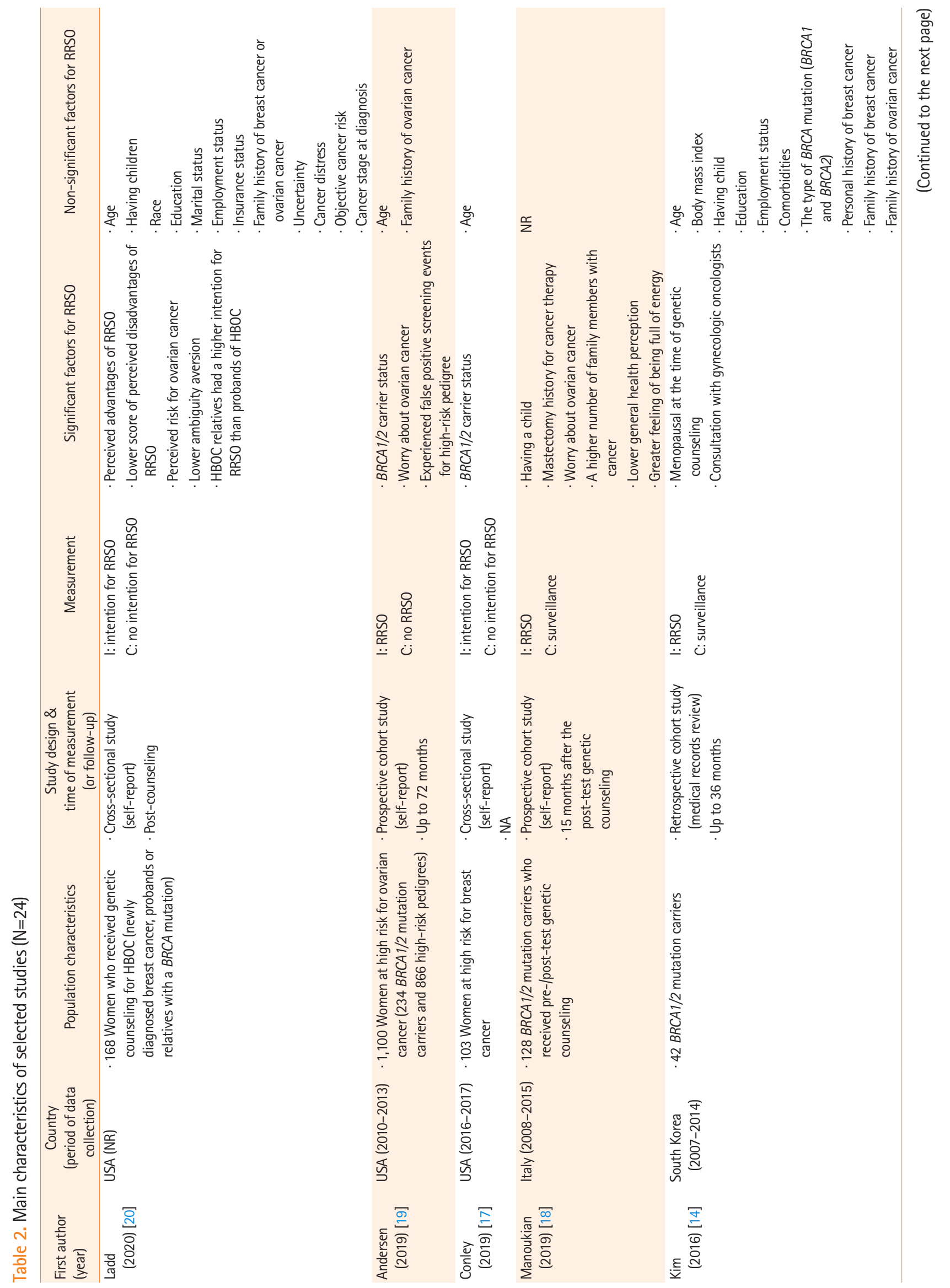




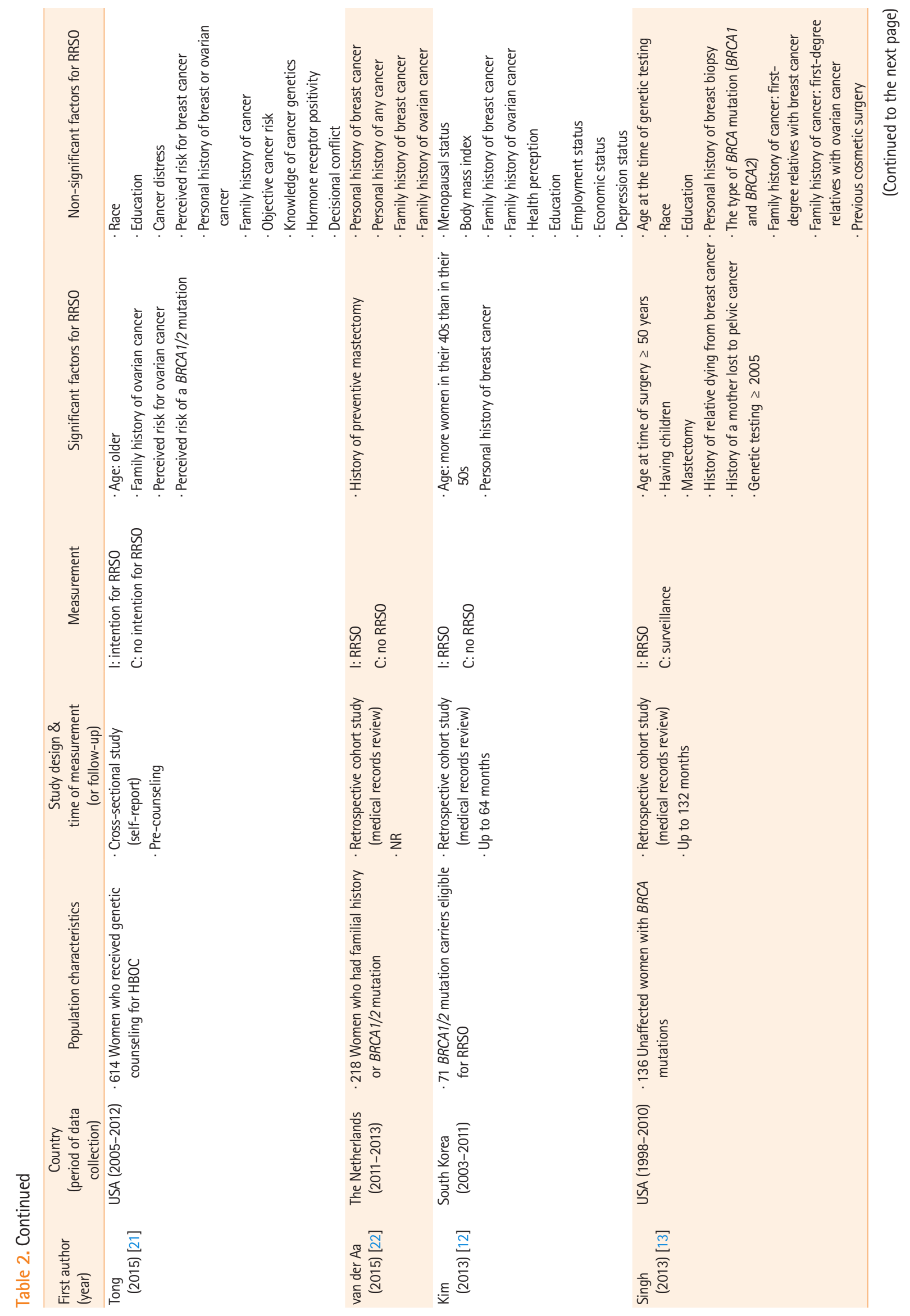




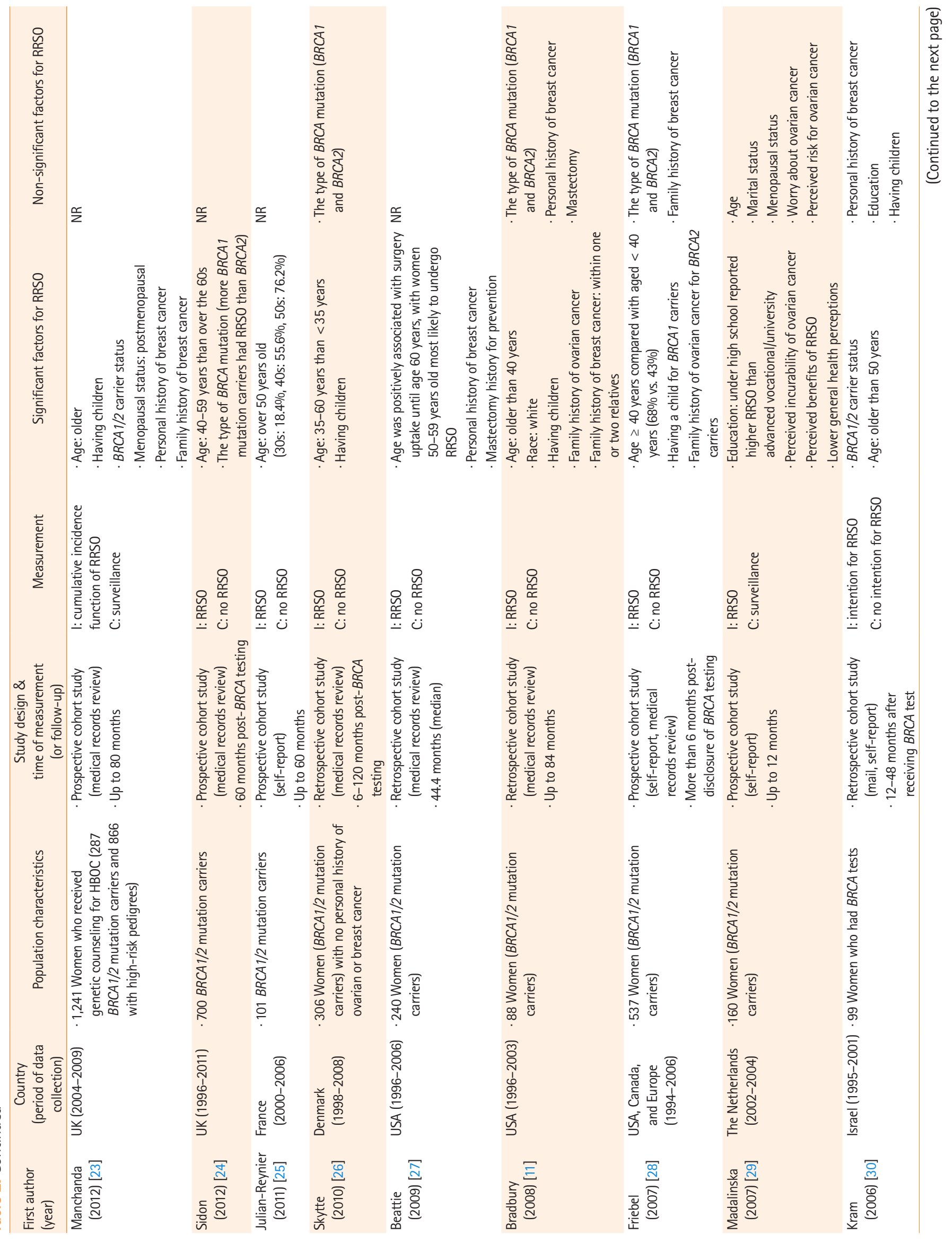




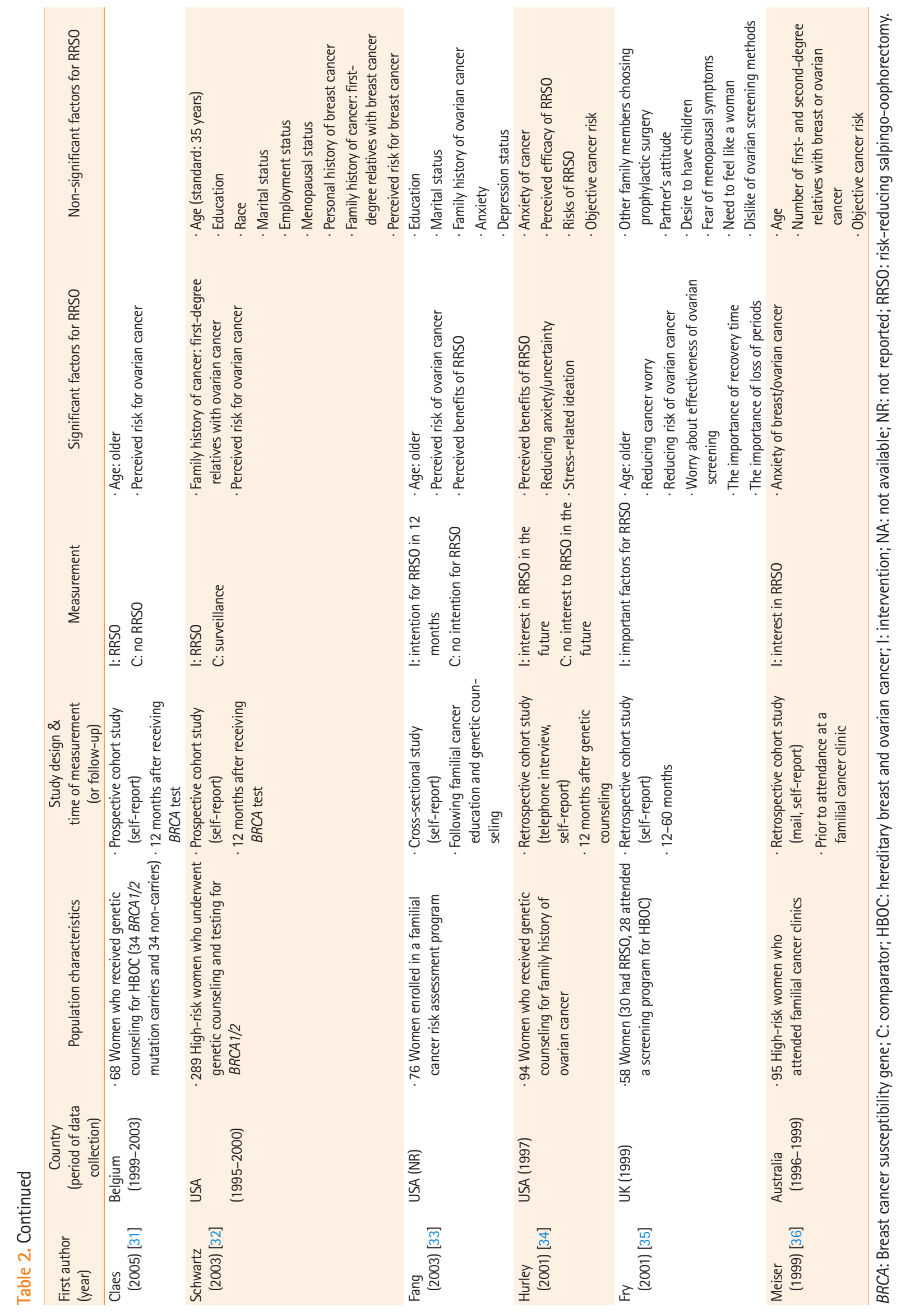




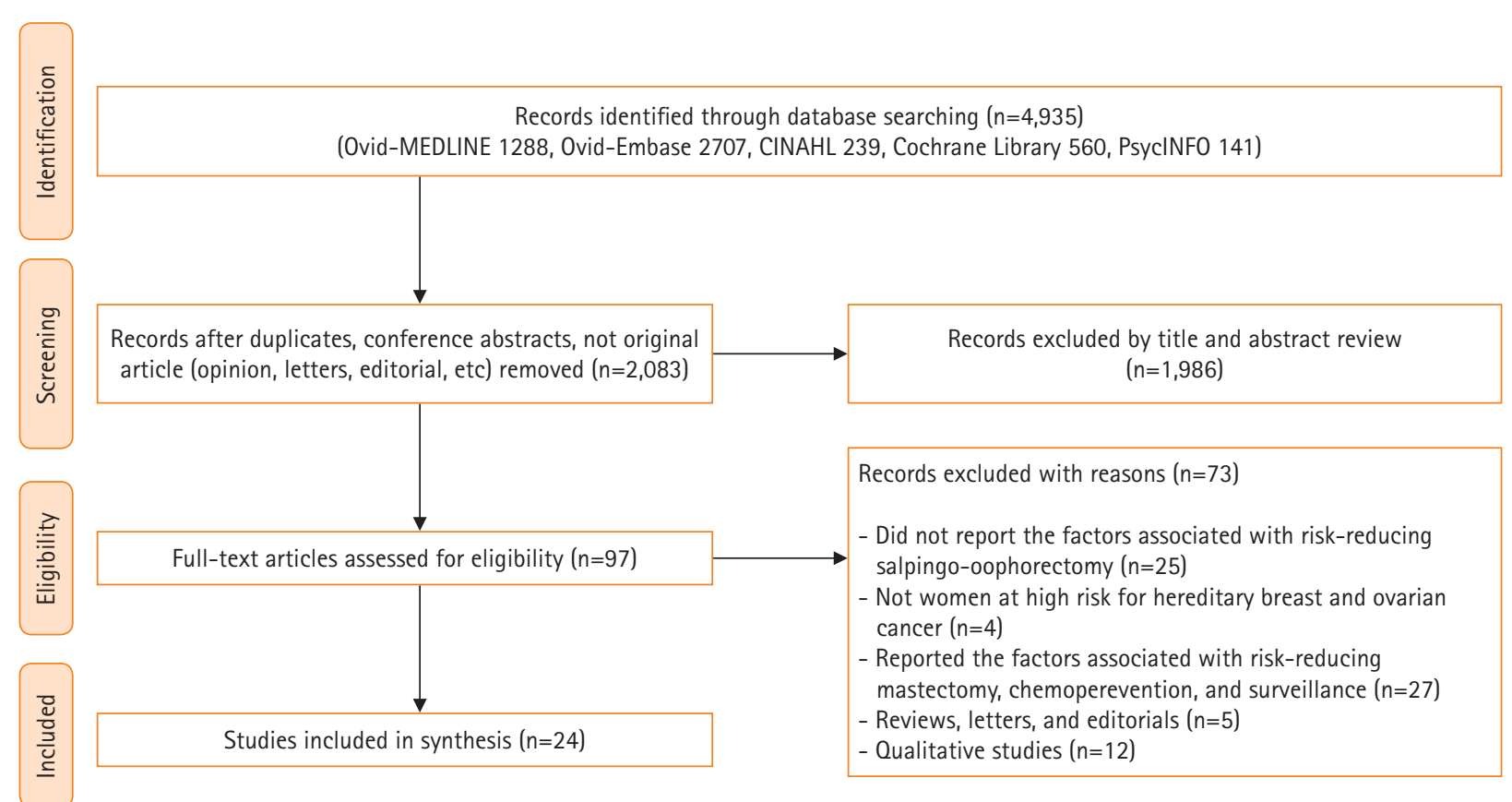

Figure 1. Flow diagram of study selection.

[+], High; [-], Low; [?], unclear

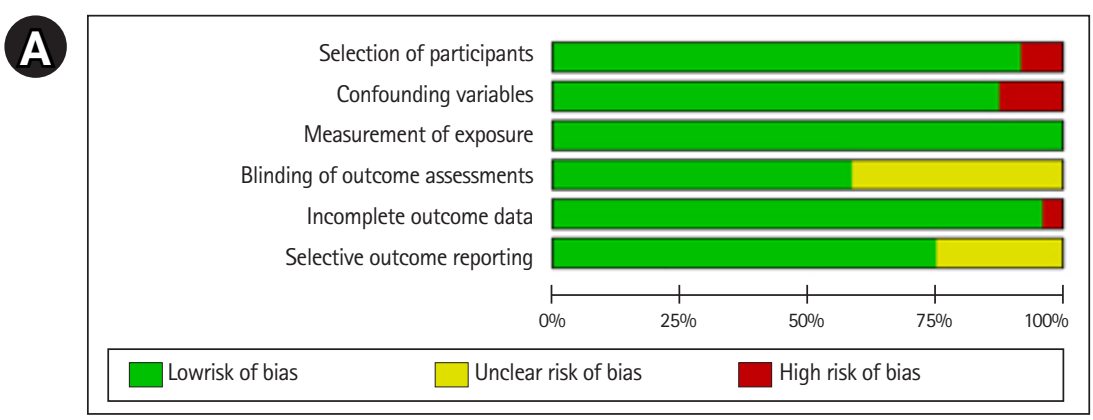

B

Figure 2. Risk-of-bias graph. (A) Risk of bias summary. (B) Risk of bias for selected studies.

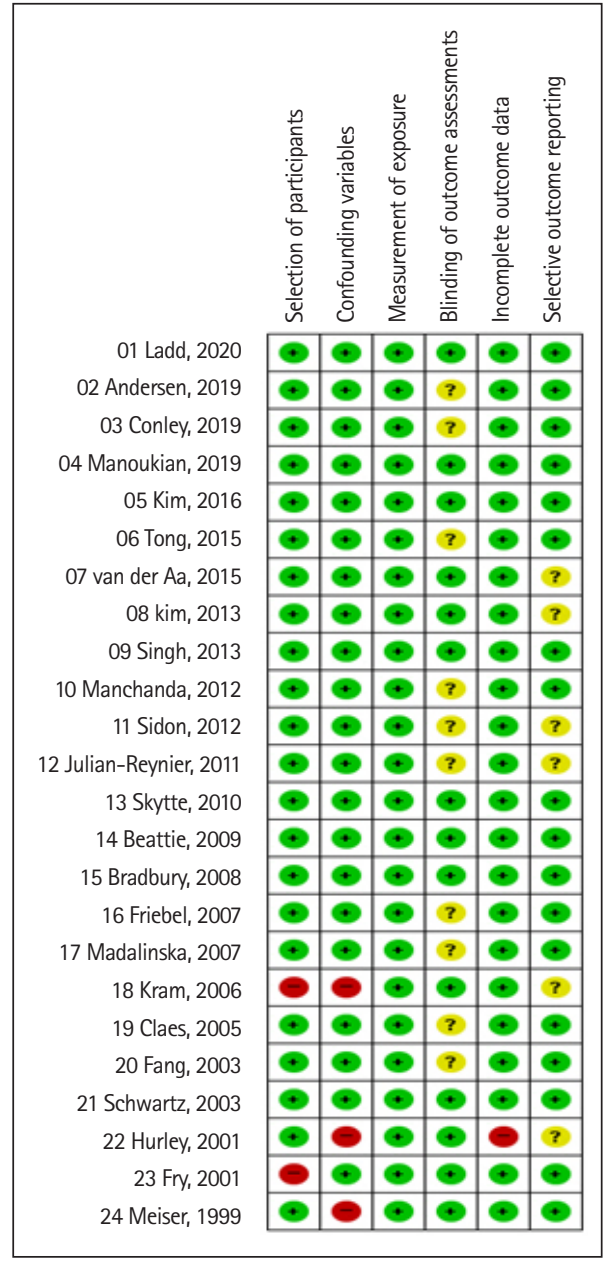


Table 3. Factors associated with the decision to undergo RRSO among women at high risk for hereditary breast and ovarian cancer

\begin{tabular}{|c|c|c|c|c|c|}
\hline \multirow{2}{*}{ Factor } & & \multicolumn{2}{|c|}{ Significant factors for RRSO } & \multicolumn{2}{|c|}{ Non-significant factors for RRSO } \\
\hline & & No. of articles ${ }^{\dagger}$ & References & No. of articles ${ }^{\dagger}$ & References \\
\hline \multirow[t]{6}{*}{ Demographic factors } & - Age & $13 / 19$ & {$[11-13,21,23-26,28,30,31,33,35]$} & $6 / 19$ & {$[17,19,20,29,32,36]$} \\
\hline & - Marital status & $1 / 5$ & [11] & $4 / 5$ & {$[20,29,32,33]$} \\
\hline & · Having child(ren) & $3 / 5$ & {$[11,23,26]$} & $2 / 5$ & {$[20,30]$} \\
\hline & - Employment status & $0 / 4$ & Not reported & $4 / 4$ & {$[12,14,20,32]$} \\
\hline & - Education level & $1 / 9$ & [29] & $8 / 9$ & {$[12-14,20,21,30,32,33]$} \\
\hline & - Race & $1 / 5$ & [11] & $4 / 5$ & {$[13,20,21,32]$} \\
\hline \multirow[t]{5}{*}{ Clinical factors } & - BRCA1/2 carrier status & $4 / 4$ & {$[17,19,23,30]$} & $0 / 4$ & Not reported \\
\hline & $\begin{array}{l}\text { - The type of } B R C A \text { (BRCA1 vs. } \\
\text { BRCA2) }\end{array}$ & $1 / 6$ & {$[24]$} & $5 / 6$ & {$[11,13,14,26,28]$} \\
\hline & - Menopausal status & $2 / 5$ & {$[14,23]$} & $3 / 5$ & {$[12,29,32]$} \\
\hline & - Mastectomy & $4 / 5$ & {$[13,18,22,27]$} & $1 / 5$ & [11] \\
\hline & $\begin{array}{l}\text { Personal history of breast } \\
\text { cancer }\end{array}$ & $3 / 9$ & {$[12,23,27]$} & $6 / 9$ & {$[11,12,21,22,30,32]$} \\
\hline \multirow[t]{2}{*}{ Family history of cancer } & $\begin{array}{l}\text { - Family history of breast } \\
\text { cancer }\end{array}$ & $2 / 11$ & {$[11,23]$} & $9 / 11$ & {$[12-14,20-22,28,32,36]$} \\
\hline & $\begin{array}{l}\text { - Family history of ovarian } \\
\text { cancer }\end{array}$ & $4 / 12$ & {$[11,21,28,32]$} & $8 / 12$ & {$[12-14,19,20,22,33,36]$} \\
\hline \multirow[t]{8}{*}{ Psychological factors } & $\begin{array}{l}\text { Perceived risk/worry/anxiety } \\
\text { for ovarian cancer }\end{array}$ & $11 / 12$ & {$[18-21,29,31-36]$} & $1 / 12$ & [29] \\
\hline & $\begin{array}{l}\text { - Perceived risk for breast } \\
\text { cancer }\end{array}$ & $0 / 2$ & Not reported & $2 / 2$ & {$[21,32]$} \\
\hline & $\begin{array}{l}\text { - Perceived advantages of } \\
\text { RRSO }\end{array}$ & $4 / 4$ & {$[20,29,33,34]$} & $0 / 4$ & Not reported \\
\hline & $\begin{array}{l}\text { Perceived disadvantages of } \\
\text { RRSO }\end{array}$ & $1 / 2$ & [20] & $1 / 2$ & {$[34]$} \\
\hline & - General health perceptions & $1 / 2$ & [29] & $1 / 2$ & [12] \\
\hline & - Cancer distress & $0 / 2$ & Not reported & $2 / 2$ & {$[20,21]$} \\
\hline & - Anxiety & $0 / 2$ & Not reported & $2 / 2$ & {$[32,34]$} \\
\hline & - Depression & $0 / 2$ & Not reported & $2 / 2$ & {$[12,33]$} \\
\hline Objective cancer risk & & $0 / 4$ & & $4 / 4$ & {$[20,21,34,36]$} \\
\hline
\end{tabular}

BRCA: Breast cancer susceptibility gene; RRSO: risk-reducing salpingo-oophorectomy.

${ }^{\dagger}$ Reported in articles/total articles.

ies $[17,20,21,30,33,36], 11 \%$ to $61.6 \%$ of women at high risk for HBOC intended to undergo RRSO in the future (mean, $41.6 \%$; 481 of 1,155 women). In 15 studies [11-14,18,22-29,31,32], $46.2 \%$ of women received RRSO (range, $23.4 \%-87.2 \% ; 1,830$ of 3,960 women (Table 4).

Four articles $[14,12,26,27]$ reported the length of time that elapsed between the BRCA test and RRSO (Table 4). Of the three articles that studied Koreans [12,14] and Americans [27], the mean time to decide was 2 to 7.3 months. Meanwhile, a Danish article [26] reported that it took 34 months to decide, and a Korean article [12] reported that the maximum time to decide was 64 months. In two Korean articles on BRCA carriers $[12,14]$, the proportion of patients who received RRSO within 1 year after receiving a genetic consultation was reported to be high, at $85.7 \%$ [12] and $86.4 \%$ [14], respectively.

\section{Discussion}

The goal of this study was to identify significant factors affecting RRSO decision-making among women at high risk of HBOC and to explore the uptake rate and decision timing for RRSO. Among the reviewed articles, 13 [11-13,21,23-26,28,30,31,33,35] suggested that older age was associated with the decision to undergo RRSO. Although six studies [17,19,20,29,32,36] did not find that age was significant, those studies analyzed age as a continuous variable; therefore, they failed to determine which age group received RRSO more. In this review, women in their 40s and 50s were more likely to undergo RRSO than other age groups in four studies $[12,13,24,25]$, and the studies that analyzed age groups were more reliable than those that examined age as a continuous variable. Therefore, age is considered to 
Table 4. RRSO rate and timing among women at high risk for HBOC

\begin{tabular}{|c|c|c|c|c|}
\hline First author /publication year & Country & Follow-up (month) & RRSO, n (\%) & Timing for RRSO (month), mean (range) \\
\hline \multicolumn{5}{|c|}{ Intention for RRSO in women at high risk for $\mathrm{HBOC}$} \\
\hline Ladd (2020) [20] & USA & NR & 103/168 (61.3) & NR \\
\hline Conley (2019) [17] & USA & NR & $11 / 103(10.7)$ & NR \\
\hline Tong (2015) [21] & USA & NR & $261 / 614(42.5)$ & NR \\
\hline Kram (2006) [30] & Israel & $12-48$ & $61 / 99(61.6)$ & NR \\
\hline Fang (2003) [33] & USA & NR & $26 / 76(34.2)$ & NR \\
\hline Meiser (1999) [36] & Australia & NR & 19/95 (20.0) & NR \\
\hline Total & & & $481 / 1,155(41.6)$ & \\
\hline \multicolumn{5}{|c|}{ RRSO in women at high risk for $\mathrm{HBOC}$} \\
\hline Manoukian (2019) [18] & Italy & 15 & $55 / 128(43.0)$ & NR \\
\hline Kim (2016) [14] & South Korea & 36 & $22 / 42(52.4)$ & $7.3(0.6-33.9)$ \\
\hline van der Aa (2015) [22] & The Netherlands & NR & $190 / 218(87.2)$ & NR \\
\hline $\operatorname{Kim}(2013)$ [12] & South Korea & 64 & $21 / 71(29.6)$ & $2(0-64)$ \\
\hline Singh (2013) [13] & USA & NR & $71 / 136(52.2)$ & NR \\
\hline Manchanda (2012) [23] & UK & 80 & $265 / 1,133(23.4)$ & NR \\
\hline Sidon (2012) [24] & UK & 60 & $309 / 700(44.1)$ & NR \\
\hline Julian-Reynier (2011) [25] & France & 60 & 43/101 (42.6) & NR \\
\hline Skytte (2010) [26] & Denmark & $6-120$ & 218/306 (71.2) & 34 \\
\hline Beattie (2009) [27] & USA & $6-120$ & $122 / 240(50.8)$ & 4 \\
\hline Bradbury (2008) [11] & USA & 84 & $62 / 88(70.5)$ & NR \\
\hline Friebel (2007) [28] & North America, EU & $\geq 6$ & $297 / 537(55.3)$ & NR \\
\hline Madalinska (2007) [29] & Netherlands & 12 & $118 / 160(73.8)$ & NR \\
\hline Claes (2005) [31] & Belgium & 12 & $16 / 21(75.0)$ & NR \\
\hline Schwartz (2003) [32] & USA & 12 & $21 / 79(26.6)$ & NR \\
\hline Total & & & $1,830 / 3,960(46.2)$ & \\
\hline
\end{tabular}

HBOC: Hereditary breast and ovarian cancer; NR: not reported; RRSO: risk-reducing salpingo-oophorectomy.

have a significant influence on decision-making about RRSO.

Although our study and the previous systematic review [7] did not confirm whether having child(ren) $[11,20,23,26,30]$ or menopause $[12,14,23,29,33]$ affected RRSO decisions, childbirth and menopause status are important variables in the decision-making process for RRSO [7]. This is because women at high risk for HBOC fear surgical-related menopause [7], and fertility is important for women who want to become pregnant. Therefore, qualitative studies that explore how fertility and menopause affect decision-making through in-depth interviews would facilitate a deeper understanding of this issue.

In this review, a personal history of mastectomy was a significant factor affecting RRSO in $80.0 \%$ of the articles, but a history of breast cancer was not a significant factor in $40.0 \%$ of the studies. A previous systematic review also showed that women with breast cancer tended to select RRM more frequently than RRSO [7]. Further research is needed to examine whether breast cancer history is associated with RRM, and if having a mastectomy affects decision-making about RRSO.

Most of the selected studies showed that a family history of breast cancer $(81.8 \%)$ and ovarian cancer $(66.7 \%)$ were not associated with having RRSO. This result is supported by a systematic review reporting that RRM was more strongly affected than RSSO by family cancer history [7]. Therefore, a family history of ovarian/or breast cancer is assumed to be a more important factor in determining RRM than RRSO. In one article [13], RRSO was more likely to be chosen if a mother or relative had died from breast or ovarian cancer than simply having a family cancer history. Furthermore, Howard et al. [7] reported that RRM was more likely to be chosen based on experiences of first-degree relatives, especially mothers and sisters, rather than of having a family history of ovarian/breast cancer. Therefore, future studies should analyze the death of a close family member from cancer, as distinct from a family history of cancer.

Perceived risk of cancer is a well-known factor contributing to the choice to undergo risk-reducing surgery among women at high risk for HBOC $[5,7,37]$. Our study found that the perceived risk of ovarian cancer was the main motivation for choosing to undergo RRSO. However, the mechanism underlying cancer risk perception is still unknown [38]. Four articles 
$[5,19,23,30]$ reported that $B R C A$ carriers chose RRSO more frequently than non-carriers, which was an expected result. Although Padamsee et al. [5] suggested that the perception of RRSO could vary depending on the type of $B R C A$ mutation, in this study there was no evidence that the type of $B R C A$ mutation affected decision-making about RRSO $[11,13,14,26,28]$. Therefore, in-depth studies are needed to determine whether there are differences in the RRSO decision-making process depending on the BRCA mutation type [5].

A systematic review [7] found that psychological factors affected decisions about RRM, but we could not confirm whether psychological stability (e.g., cancer-related distress, anxiety, and depression) affected RRSO decision-making in this study. Therefore, further studies are needed to identify differences in psychological motivations for decisions about RRSO and RRM.

Previous qualitative studies showed that family factors were related to RRSO $[7,39]$, and a systematic review found that spouses, family/friends, and doctors' recommendations influenced the choice to undergo RRM [38]. However, we could not determine whether these factors were explored in quantitative studies related to RRSO. These gaps may suggest that family and interpersonal factors in RRSO decision-making have not been explored. However, family, friends, and communities influence the information obtained and the decision-making process. Therefore, further research is needed to identify the impact of these factors and to integrate the factors reported in qualitative studies.

In this review, objective cancer risk was not related to the decision to undergo RRSO. This result implies that women decide to undergo RRSO to reduce anxiety based on the perceived risk of ovarian cancer [40], rather than on objective information. In addition, the effect of genetic testing on RRSO decisions has not been reported to a sufficient extent. Therefore, it is necessary to confirm whether fully-informed decision-making is happening in the clinical setting.

The uptake rate of RRSO varied from $11 \%$ to $87.2 \%$ across the selected articles in this review. Among those who opted for RRSO, Koreans were younger than Europeans [12,13,25], and $71.2 \%$ to $87.2 \%$ of Danish [26] and Dutch [22,29] women chose RRSO, which was a higher rate than that of women in other countries. This study also showed that Danish women took a longer time to make decisions than Koreans and Americans. These results imply that socio-cultural factors and national health care systems may affect RRSO decisions. This is supported by Padamsee et al. [5], who suggested that geographical differences, which may be a proxy for differences in health care infrastructure and cultural contexts, influence RRSO decisions.
Therefore, further research is needed to examine how sociocultural factors and health care delivery systems affect RRSO decision-making and surgical timing.

The generalizability of the results of this systematic review is limited because we did not review the factors associated with RRSO from qualitative research. Nevertheless, this study is meaningful in that it provides fundamental information regarding factors affecting RRSO decisions based on current evidence. In particular, we found that the perceived risk of ovarian cancer, older age, and being a BRCA carrier are major factors affecting RRSO decision-making.

Based on the results of this study, we suggest the following: (1) considering that the decision process of RRSO is complex and involves various factors, it is necessary to identify how family factors, socio-cultural characteristics, and healthcare systems affect the decision process; (2) further studies are needed to confirm the significance of factors that have been reported in a few studies or have shown contradictory results across articles; and (3) interventions should be developed based on information about objective cancer risk.

\section{ORCID}

Sun-young Park, https://orcid.org/0000-0003-3560-8369

Youlim Kim, https://orcid.org/0000-0002-4477-2489

Sue Kim, https://orcid.org/0000-0003-3785-2445

\section{Authors' contributions}

Conceived and designed the research: Park S, Kim S; Searched the data: Park S; Contributed selection and quality assessment of the literature, Analyzed the data: Park S, Kim Y; Writingoriginal draft: all authors; Writing-review \& editing: all authors.

\section{Conflict of interest}

Sue Kim has been editor of the Korean Journal of Women Health Nursing since January 2020. She was not involved in the review process of this manuscript. Otherwise, there was no conflict of interest.

\section{Funding}

None. 


\section{Data availability}

The datasets are available from the corresponding author on reasonable request.

\section{Acknowledgments}

We thank Gayoung Shin, MS for contributing to the selection of the articles.

\section{Supplementary materials}

Further details on supplementary materials are presented online (available at: https://doi.org/10.4069/kjwhn.2020.11.19).

\section{References}

1. Hartmann LC, Lindor NM. The role of risk-reducing surgery in hereditary breast and ovarian cancer. N Engl J Med. 2016;374(5):454-468. https://doi.org/10.1056/NEJMra1 503523

2. Petrucelli N, Daly MB, Feldman GL. Hereditary breast and ovarian cancer due to mutations in BRCA1 and BRCA2. Genet Med. 2010;12(5):245-259. https://doi.org/10.1097/ GIM.0b013e3181d38f2f

3. Kang E, Seong MW, Park SK, Lee JW, Lee J, Kim LS, et al. The prevalence and spectrum of BRCA1 and BRCA2 mutations in Korean population: recent update of the Korean Hereditary Breast Cancer (KOHBRA) study. Breast Cancer Res Treat. 2015;151(1):157-168. https://doi.org/10.1007/s10549015-3377-4

4. Rebbeck TR, Kauff ND, Domchek SM. Meta-analysis of risk reduction estimates associated with risk-reducing salpingo-oophorectomy in BRCA1 or BRCA2 mutation carriers. J Natl Cancer Inst. 2009;101(2):80-87. https://doi.org/10.1093/ jnci/djn442

5. Padamsee TJ, Wills CE, Yee LD, Paskett ED. Decision making for breast cancer prevention among women at elevated risk. Breast Cancer Res. 2017;19(1):34. https://doi.org/10.1186/s13058-0170826-5

6. Meadows R, Padamsee TJ, Paskett ED. Distinctive psychological and social experiences of women choosing prophylactic oophorectomy for cancer prevention. Health Care Women Int. 2018;39(5):595-616. https://doi.org/10.1080/07399332 .2018 .1424855

7. Howard AF, Balneaves LG, Bottorff JL. Women's decision making about risk-reducing strategies in the context of he- reditary breast and ovarian cancer: a systematic review. J Genet Couns. 2009;18(6):578-597. https://doi.org/10.1007/ s10897-009-9245-9

8. Hickey M, Rio I, Trainer A, Marino JL, Wrede CD, Peate M. What information do healthcare professionals need to inform premenopausal women about risk-reducing salpingo-oophorectomy? Menopause. 2020;27(1):20-25. https://doi.org/10. 1097/GME.0000000000001421

9. Cherry C, Ropka M, Lyle J, Napolitano L, Daly MB. Understanding the needs of women considering risk-reducing salpingo-oophorectomy. Cancer Nurs. 2013;36(3):E33-E38. https:// doi.org/10.1097/NCC.0b013e3182642cb5

10. Rosenberg SM, Greaney ML, Patenaude AF, Sepucha KR, Meyer ME, Partridge AH. "I don't want to take chances.": a qualitative exploration of surgical decision making in young breast cancer survivors. Psychooncology. 2018;27(6):15241529. https://doi.org/10.1002/pon.4683

11. Bradbury AR, Ibe CN, Dignam JJ, Cummings SA, Verp M, White MA, et al. Uptake and timing of bilateral prophylactic salpingo-oophorectomy among BRCA1 and BRCA2 mutation carriers. Genet Med. 2008;10(3):161-166. https://doi. org/10.1097/GIM.0b013e318163487d

12. Kim D, Kang E, Hwang E, Sun Y, Hwang Y, Yom CK, et al. Factors affecting the decision to undergo risk-reducing salpingo-oophorectomy among women with BRCA gene mutation. Fam Cancer. 2013;12(4):621-628. https://doi.org/10. 1007/s10689-013-9625-Z

13. Singh K, Lester J, Karlan B, Bresee C, Geva T, Gordon O. Impact of family history on choosing risk-reducing surgery among BRCA mutation carriers. Am J Obstet Gynecol. 2013;208(4):329.e1-329.e3296. https://doi.org/10.1016/j.ajog. 2013.01.026

14. Kim SI, Lim MC, Lee DO, Kong SY, Seo SS, Kang S, et al. Uptake of risk-reducing salpingo-oophorectomy among female BRCA mutation carriers: experience at the National Cancer Center of Korea. J Cancer Res Clin Oncol. 2016;142(1):333340. https://doi.org/10.1007/s00432-015-2051-x

15. Moher D, Liberati A, Tetzlaff J, Altman DG; PRISMA Group. Preferred reporting items for systematic reviews and meta-analyses: the PRISMA statement. PLoS Med. 2009;6(7):e1000097. https://doi.org/10.1371/journal.pmed.1000097

16. Kim SY, Park JE, Lee YJ, Seo HJ, Sheen SS, Hahn S, et al. Testing a tool for assessing the risk of bias for nonrandomized studies showed moderate reliability and promising validity. J Clin Epidemiol. 2013;66(4):408-414. https://doi.org/10.1016/ j.jclinepi.2012.09.016

17. Conley CC, Agnese DM, Vadaparampil ST, Andersen BL. 
Factors associated with intentions for breast cancer risk management: does risk group matter? Psychooncology. 2019; 28(5):1119-1126. https://doi.org/10.1002/pon.5066

18. Manoukian S, Alfieri S, Bianchi E, Peissel B, Azzollini J, Borreani C. Risk-reducing surgery in BRCA1/BRCA2 mutation carriers: are there factors associated with the choice? Psychooncology. 2019;28(9):1871-1878. https://doi.org/10.1002/ pon.5166

19. Andersen MR, Karlan BY, Drescher CW, Paley P, Hawley S, Palomares M, et al. False-positive screening events and worry influence decisions about surgery among high-risk women. Health Psychol. 2019;38(1):43-52. https://doi.org/10.1037/ hea0000647

20. Ladd MK, Peshkin BN, Senter L, Baldinger S, Isaacs C, Segal $\mathrm{H}$, et al. Predictors of risk-reducing surgery intentions following genetic counseling for hereditary breast and ovarian cancer. Transl Behav Med. 2020;10(2):337-346. https://doi. org/10.1093/tbm/iby101

21. Tong A, Kelly S, Nusbaum R, Graves K, Peshkin BN, Valdimarsdottir $\mathrm{HB}$, et al. Intentions for risk-reducing surgery among high-risk women referred for BRCA1/BRCA2 genetic counseling. Psychooncology. 2015;24(1):33-39. https://doi. org/10.1002/pon.3560

22. van der Aa JE, Hoogendam JP, Butter ES, Ausems MG, Verheijen RH, Zweemer RP. The effect of personal medical history and family history of cancer on the uptake of risk-reducing salpingo-oophorectomy. Fam Cancer. 2015;14(4):539544. https://doi.org/10.1007/s10689-015-9827-7

23. Manchanda R, Burnell M, Abdelraheim A, Johnson M, Sharma A, Benjamin E, et al. Factors influencing uptake and timing of risk reducing salpingo-oophorectomy in women at risk of familial ovarian cancer: a competing risk time to event analysis. BJOG. 2012;119(5):527-536. https://doi.org/10. 1111/j.1471-0528.2011.03257.x

24. Sidon L, Ingham S, Clancy T, Clayton R, Clarke A, Jones EA, et al. Uptake of risk-reducing salpingo-oophorectomy in women carrying a BRCA1 or BRCA2 mutation: evidence for lower uptake in women affected by breast cancer and older women. Br J Cancer. 2012;106(4):775-779. https://doi.org/ 10.1038/bjc.2011.573

25. Julian-Reynier C, Mancini J, Mouret-Fourme E, Gauthier-Villars M, Bonadona V, Berthet $\mathrm{P}$, et al. Cancer risk management strategies and perceptions of unaffected women 5 years after predictive genetic testing for BRCA1/2 mutations. Eur J Hum Genet. 2011;19(5):500-506. https://doi.org/10. 1038/ejhg.2010.241

26. Skytte AB, Gerdes AM, Andersen MK, Sunde L, Brøn-
dum-Nielsen K, Waldstrøm M, et al. Risk-reducing mastectomy and salpingo-oophorectomy in unaffected BRCA mutation carriers: uptake and timing. Clin Genet. 2010;77(4):342349. https://doi.org/10.1111/j.1399-0004.2009.01329.x

27. Beattie MS, Crawford B, Lin F, Vittinghoff E, Ziegler J. Uptake, time course, and predictors of risk-reducing surgeries in BRCA carriers. Genet Test Mol Biomarkers. 2009;13(1):5156. https://doi.org/10.1089/gtmb.2008.0067

28. Friebel TM, Domchek SM, Neuhausen SL, Wagner T, Evans DG, Isaacs C, et al. Bilateral prophylactic oophorectomy and bilateral prophylactic mastectomy in a prospective cohort of unaffected BRCA1 and BRCA2 mutation carriers. Clin Breast Cancer. 2007;7(11):875-882. https://doi.org/10.3816/ CBC.2007.n.053

29. Madalinska JB, van Beurden M, Bleiker EM, Valdimarsdottir HB, Lubsen-Brandsma L, Massuger LF, et al. Predictors of prophylactic bilateral salpingo-oophorectomy compared with gynecologic screening use in BRCA1/2 mutation carriers. J Clin Oncol. 2007;25(3):301-307. https://doi.org/10.1200/ JCO.2006.07.4922

30. Kram V, Peretz T, Sagi M. Acceptance of preventive surgeries by Israeli women who had undergone BRCA testing. Fam Cancer. 2006;5(4):327-335. https://doi.org/10.1007/s10689006-0002-Z

31. Claes E, Evers-Kiebooms G, Decruyenaere M, Denayer L, Boogaerts A, Philippe K, et al. Surveillance behavior and prophylactic surgery after predictive testing for hereditary breast/ ovarian cancer. Behav Med. 2005;31(3):93-105. https://doi. org/10.3200/BMED.31.3.93-106

32. Schwartz MD, Kaufman E, Peshkin BN, Isaacs C, Hughes C, DeMarco T, et al. Bilateral prophylactic oophorectomy and ovarian cancer screening following BRCA1/BRCA2 mutation testing. J Clin Oncol. 2003;21(21):4034-4041. https://doi. org/10.1200/JCO.2003.01.088

33. Fang CY, Miller SM, Malick J, Babb J, Hurley KE, Engstrom $\mathrm{PF}$, et al. Psychosocial correlates of intention to undergo prophylactic oophorectomy among women with a family history of ovarian cancer. Prev Med. 2003;37(5):424-431. https://doi. org/10.1016/s0091-7435(03)00163-4

34. Hurley KE, Miller SM, Costalas JW, Gillespie D, Daly MB. Anxiety/uncertainty reduction as a motivation for interest in prophylactic oophorectomy in women with a family history of ovarian cancer. J Womens Health Gend Based Med. 2001;10(2):189-199. https://doi.org/10.1089/152460901300039566

35. Fry A, Rush R, Busby-Earle C, Cull A. Deciding about prophylactic oophorectomy: what is important to women at increased risk of ovarian cancer? Prev Med. 2001;33(6):578- 
585. https://doi.org/10.1006/pmed.2001.0924

36. Meiser B, Butow P, Barratt A, Friedlander M, Gattas M, Kirk J, et al. Attitudes toward prophylactic oophorectomy and screening utilization in women at increased risk of developing hereditary breast/ovarian cancer. Gynecol Oncol. 1999; 75(1):122-129. https://doi.org/10.1006/gyno.1999.5544

37. Glassey R, Ives A, Saunders C, Musiello T. Decision making, psychological wellbeing and psychosocial outcomes for high risk women who choose to undergo bilateral prophylactic mastectomy - a review of the literature. Breast. 2016;28:130135. https://doi.org/10.1016/j.breast.2016.05.012

38. Ager B, Butow P, Jansen J, Phillips KA, Porter D; CPM DA Advisory Group. Contralateral prophylactic mastectomy
(CPM): a systematic review of patient reported factors and psychological predictors influencing choice and satisfaction. Breast. 2016;28:107-120. https://doi.org/10.1016/j.breast. 2016.04.005

39. Sun S, Li ST, Ngeow J. Factors shaping at-risk individuals' decisions to undergo genetic testing for cancer in Asia. Health Soc Care Community. 2020;28(5):1569-1577. https://doi.org/ 10.1111/hsc.12981

40. Haroun I, Graham T, Poll A, Sun P, Hill K, Weitzner E, et al. Reasons for risk-reducing mastectomy versus MRI-screening in a cohort of women at high hereditary risk of breast cancer. Breast. 2011;20(3):254-258. https://doi.org/10.1016/j.breast. 2011.01.006 